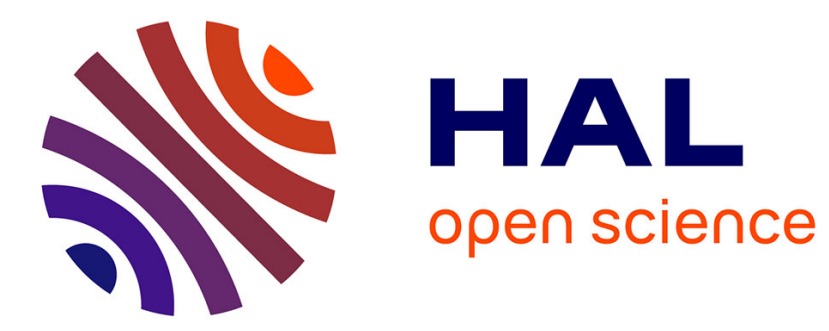

\title{
Débat autour de Capital Rules. The Construction of Global Finance de Rawi Abdelal, Cambridge, MA: Harvard University Press, 2009
}

\author{
Douglas j. Forsyth, Léonard Laborie, Rawi Abdelal
}

\section{- To cite this version:}

Douglas j. Forsyth, Léonard Laborie, Rawi Abdelal. Débat autour de Capital Rules. The Construction of Global Finance de Rawi Abdelal, Cambridge, MA: Harvard University Press, 2009. Monde(s). Histoire, Espaces, Relations, 2018, 13 (1), pp.155-171. 10.3917/mond1.181.0155 . hal-02474283

HAL Id: hal-02474283

https://cnrs.hal.science/hal-02474283

Submitted on 11 Feb 2020

HAL is a multi-disciplinary open access archive for the deposit and dissemination of scientific research documents, whether they are published or not. The documents may come from teaching and research institutions in France or abroad, or from public or private research centers.
L'archive ouverte pluridisciplinaire HAL, est destinée au dépôt et à la diffusion de documents scientifiques de niveau recherche, publiés ou non, émanant des établissements d'enseignement et de recherche français ou étrangers, des laboratoires publics ou privés. 
Le texte ci-dessous est ma contribution personnelle à l'article ainsi référencé : Douglas J. Forsyth, Léonard Laborie, Rawi Abdelal, «Débat autour de Capital Rules. The Construction of Global Finance de Rawi Abdelal, Cambridge, MA: Harvard University Press, 2009 », Monde(s) 2018/1 (N 13), p. 155-171.

DOI 10.3917/mond1.181.0155

Note d'édition : la note infrapaginale 5 annonce la parution d'un livre. Ce dernier est désormais paru sous le titre: Georges Saunier (dir.), Mitterrand, les années d'alternances. 1984-1986 / 1986-1988, Nouveau Monde, 2019.

\section{Léonard Laborie \\ CNRS UMR SIRICE, Paris}

Publié pour la première fois en 2007, au terme d'une enquête d'une dizaine d'années entamée au moment de la crise financière asiatique, ce livre porte sur les règles internationales qui gouvernent les flux de capitaux transfrontaliers. Sa thèse martelée de bout en bout prend la forme d'un diptyque : d'une part ces règles ont été considérablement assouplies, libéralisées, entre la fin des années 1980 et la fin des années 1990, d'autre part c'est aux Européens, et aux Français et aux Allemands en particulier qu'on doit cette libéralisation. La première partie de cette thèse est sans doute moins novatrice que la seconde, qui se veut même provocatrice ou « révisionniste» selon les mots de l'auteur. Elle revient en effet sur l'opinion commune («conventional wisdom») parmi les acteurs et les commentateurs selon laquelle la responsabilité première de cette transformation revient aux Etats-Unis et à leur complexe politico-financier et académique - le Trésor, Wall Street et la pensée néolibérale. On peut se demander toutefois si ce qui est présenté comme l'opinion commune n'est pas en réalité une opinion typiquement nord-américaine, témoignant d'une vision du monde précisément en ligne avec une approche unilatérale de ces questions par les dirigeants et les opérateurs financiers de ce pays. En Europe, j'ose espérer que les débats au sein de la gauche ont fait apparaître depuis plus longtemps déjà le rôle d'une partie des socialistes français dans ce sacrifice du contrôle des mouvements de capitaux sur l'autel de l'union monétaire négociée avec les Allemands. De ce côté de l'Atlantique, les contemporains les plus critiques face à ce grand changement n'ontils pas pointé le rôle moteur des Européens en général et du couple franco-allemand en particulier ? Certains n'ont-ils pas inversement contribué à obscurcir cette réalité en blâmant pêle-mêle le néolibéralisme, la banque et les manipulations américaines ? On pourrait revenir dans cette perspective sur les discussions ravivées dans les années 1990 autour du projet de taxe sur les transactions financières internationales et sur le mouvement ATTAC en France par exemple.

Quoi qu'il en soit de cette question du cadrage de l'opinion dite commune, le grand mérite de Rawi Abdelal est de recomposer avec agilité et force de conviction un puzzle où s'assemblent des pièces jusqu'alors dispersées dans notre esprit. Ces pièces ont pour nom à la fois des organisations (le département du Trésor américain, la Commission et l'Union européennes, l'OCDE et son Comité sur les mouvements de capitaux et les transactions invisibles, le FMI), 
des entreprises (les agences de notation Standard \& Poor's ou Moody's, qui notent les États ou les entreprises cherchant à se financer sur les marchés) et des individus (en particulier les Jacques Delors, Pascal Lamy, Michel Camdessus, Henri Chavranski, cette minorité parmi les socialistes français qui tient le stylo en France et dans les organisations précitées à partir de 1983). Ces différentes pièces sont rapprochées les unes des autres, prennent sens les unes par rapport aux autres, mais ne sont jamais confondues. C'est un point clé d'une démonstration menée en neuf chapitres enlevés.

L'auteur distingue en effet deux approches de la globalisation financière. Cette distinction est présentée d'emblée, dans les deux premiers chapitres, car elle structure le livre. Il y a d'une part une approche unilatérale, celle des Etats-Unis, qui font la globalisation à leur convenance (« ad hoc globalization »). Ils y travaillent depuis les années 1950, en lien avec les agences de notation, la place de Londres et avec l'appui politique de la RFA. Il y a d'autre part une approche multilatérale, portée par les Européens depuis les années 1980. Les deux s'en prennent à la régulation poussée mise en œuvre au lendemain de la Seconde Guerre mondiale, à travers les accords de Bretton Woods et le Traité de Rome, ce dernier qui ne reconnaît que trois libertés fondamentales de circulation (des personnes, des marchandises et des services) bridant considérablement celle des capitaux (chapitre 3). Ces deux approches sont concurrentes et se succèdent dans le temps - sans que le lien causal ne soit d'ailleurs clairement discuté : qu'estce que la libéralisation impulsée par les Européens doit à celle impulsée par les Américains avant eux ? Ce sont les Européens qui ont la main au sein de la Communauté économique européenne en train de se transformer en Union européenne d'abord (1986-1992, chapitre 4), puis au sein de l'OCDE (1989-1992, chapitre 5) et enfin au FMI (1990-1999, chapitre 6) où le projet de libéralisation échoue finalement dans le contexte des crises financières en Amérique latine, en Europe et surtout en Asie. L'Union européenne et dans une moindre mesure l'OCDE se trouvent alors seules à porter le dogme d'une libéralisation totale et codifiée internationalement des flux financiers transfrontaliers, tandis que les agences de notation (chapitre 7) et les États-Unis poursuivent leur approche au cas par cas du sujet (chapitres 8 et 9 ).

Je ne ferai pas la critique attendue de la part d'un historien des techniques et de l'innovation de l'absence des ordinateurs, des télex et autres réseaux de communication dans une étude sur les marchés financiers que l'on sait pourtant très en pointe en la matière ${ }^{1}$. D'accord avec l'auteur, je pense que les techniques ne dictent pas les règles internationales qu'écrivent ensemble les experts et les gouvernements. Ce n'est pas parce que le premier câble transatlantique à fibres optiques a été posé en 1988 que la directive européenne de la même année a fixé comme cap l'interdiction du contrôle des capitaux entre pays membres et avec les pays tiers. En revanche, ces dispositifs permettent de jouer différemment avec les règles, de les pousser à leurs limites ou de les contourner, et peuvent avoir des conséquences inattendues. Sur ce point, la question

\footnotetext{
${ }^{1}$ Sur la globalisation financière du $19^{\mathrm{e}}$ siècle, la propagation des crises et l'invention de produits financiers comme les futures en lien avec les réseaux télégraphiques, voir notamment : Simone M. Müller, Heidi J.S. Tworek, «'The telegraph and the bank': on the interdependence of global communications and capitalism, 1866-1914» et Alexander Engel, «Buying time: futures trading and telegraphy in nineteenth-century global commodity markets », Journal of Global History, 10/2, 2015.
} 
que l'on peut se poser est la suivante : les décideurs ont-ils traité le sujet de la circulation de l'argent et des produits financiers à travers les frontières sans prendre en compte ces paramètres techniques ou avaient-ils conscience de leur importance pratique? Autrement dit, quelle place leur modèle interprétatif de la réalité accordait-il à la technique?

Le point de discussion qui me paraît le plus important porte sur le poids donné à ce petit groupe de décideurs ou d'animateurs français socialistes. D'un côté l'auteur me convainc de la responsabilité de ces hommes dans le cours des événements décrits. Eux-mêmes la revendiquent clairement. D'un autre côté, je ne peux m'empêcher de m'interroger sur cette focalisation de l'auteur sur eux, et eux presque seuls. Est-ce qu'à son tour elle n'obscurcit pas un pan de la réalité ? Est-ce qu'elle ne fait pas perdre de vue d'autres acteurs ? S'il est critique de l'opinion commune, l'auteur reprend à la lettre ce que disent ces acteurs dans les entretiens qu'ils lui ont accordé à propos de ce qu'ils ont fait et de la magnitude de ce qu'ils ont fait. C'étaient bien eux les chefs. Mais les chefs ne sont-ils pas aussi parfois des suiveurs ? La France a-t-elle prémédité l'arrivée de tous ces responsables à des postes clés au plan international ? Que dire du consentement des autres pays, et de l'Allemagne en particulier ? L'auteur présente certes lui-même des réponses à ces questions, notamment par son approche rationaliste de la stratégie française qui en puissance moyenne promeut une mondialisation maîtrisée, combinée à son approche constructiviste de l'établissement de la norme entre pairs. Structurée sans être surchargée sur le plan théorique, l'analyse revendique en effet un pragmatisme qui combine rationalisme et constructivisme. Face à la question de la codification de la liberté de circulation des capitaux à l'échelle internationale, elle explique les faits aussi bien par les enjeux de pouvoir que par les changements d'attitudes collectives dans la définition de ce qui est contextuellement jugé légitime, orthodoxe, en matière de contrôle des capitaux. Mais je ne serais pas étonné que des travaux cherchent à l'avenir ou aient déjà cherché à nuancer la place donnée à cette poignée d'acteurs de la même nationalité et du même bord politique. Elle est si centrale et sans aspérité qu'on finit par douter, et le doute anime l'esprit d'enquête. L'auteur est moins disert sur les acteurs allemands, pris en un bloc monolithique et presque anonyme dans la poursuite d'une politique dont ils n'auraient pas dévié et pas discuté depuis les années 1950, à savoir la suppression coûte que coûte des contrôles des capitaux pour éviter toute manipulation néfaste de la monnaie, sous peine de sanction par les marchés. Entre eux, pas de discussions, de désaccords, d'hésitations?

N'ayant pas entendu l'appel à lire et traduire ce travail « de salubrité publique » lancé par Aquilino Morelle en septembre 2011 dans Le Monde ${ }^{2}$, je remercie les coordinateurs de ce numéro de $M o n d e(s)$ de m'avoir invité à en faire le compte rendu. Je dirais d'expérience que le lecteur néophyte en matière de finance internationale sent bien qu'il ne tire pas tous les avantages de la richesse du texte. Mais l'auteur a fourni un tel travail d'écriture et de clarification de sa pensée qu'il reste accessible à tous ceux qui veulent se donner la peine (et plus encore la joie) de comprendre.

\footnotetext{
${ }^{2}$ Aquilino Morelle (alors directeur de la campagne d'Arnaud Montebourg candidat aux primaires socialistes), « La démondialisation inquiète les partisans d'un libéralisme aux abois », Le Monde, 7 septembre 2011.
} 
À mon tour, j'encourage donc la lecture d'un livre éclairant pour quiconque s'intéresse, au-delà de la question financière déterminante en soi, à ce qui fait et ce que fait l'Europe dans le monde, et accessoirement aux lignes de partage et de recomposition d'une gauche française de gouvernement appelée aujourd'hui à s'interroger sur son histoire récente. Pour qui voudrait lire l'auteur en français, je rappelle l'article publié antérieurement à ce livre consacré à ce qu'il dénomme le « consensus de Paris », par contraste avec le plus connu et décrié « consensus de Washington », construit dans l'enceinte de $1^{\prime} \mathrm{OCDE}^{3}$. Sur cette organisation, et le travail entre pairs qui y est mené, les travaux plus récents de Matthias Schmelzer s'imposent ${ }^{4}$. Sur la question du tournant libéral de la politique financière française dans la seconde moitié du premier septennat de François Mitterrand, la publication du colloque consacré aux années 19841988 du premier septennat de François Mitterrand devrait apporter de précieux compléments 5 .

À condition de ne pas la considérer comme un processus linéaire, l'histoire non seulement explique pourquoi et comment on en est arrivé là plutôt qu'ailleurs, mais tend un miroir dans lequel l'originalité de ce qui fait le présent transparait, par contraste avec le passé. Telle est la conclusion du livre, plaidoyer pour l'histoire dans la cité : il faut faire ce détour par le passé pour prendre conscience des constituants d'un présent qui n'a rien de naturel ou de donné par avance et pour toujours, et de là pour être en mesure d'agir sur lui. Aussi sommes-nous aujourd'hui désireux de lire la suite d'un livre que nous refermons en 2017 avec un décalage de dix années par rapport à son écriture - dix années riches en matière de vie financière internationale -, qui ont révélé l'ampleur des connexions transnationales et le pouvoir des agences de notation. La coexistence des deux approches de la globalisation financière, américaine et européenne, est-elle la clé de compréhension de la manière dont fut gérée internationalement la crise qui s'est ouverte en 2008, ou la crise a-t-elle plutôt fait évoluer de part et d'autre ces deux approches ? D'autres acteurs n'ont-ils pas rejoint et complexifié la scène, notamment la Chine ? L'auteur nous dira peut-être s'il travaille à l'écriture de cette suite attendue.

\footnotetext{
${ }^{3}$ Rawi Abdelal, «Le consensus de Paris : la France et les règles de la finance mondiale », Critique internationale, $\mathrm{n}^{\circ} 28,2005$, p. 87-115.

${ }^{4}$ Matthias Schmelzer, The Hegemony of Growth. The OECD and the Making of the Economic Growth Paradigm, Cambridge, Cambridge University Press, 2016.

${ }^{5}$ Serge Berstein, Frédéric Bozo et al. (dir.), Les années d'alternances. 1984-1988, Paris, Nouveau Monde, 2017 (à paraître)
} 